\title{
COMPARISON OF ISOCONAZOLE NITRATE VERSUS NYSTATIN FOR TREATMENT OF OTOMYCOSIS
}

\author{
Muhammad Ahmed Khan, Bushra Anwar*, Sumera Akram**, Attique Ahmed*, Sunarays Akhtar**, Maqbool Raza**** \\ Combined Military Hospital Mardan/National University of Medical Sciences (NUMS) Pakistan, *Combined Military Hospital Lahore/National University of \\ Medical Sciences (NUMS) Pakistan, **Mardan Medical Complex, Mardan Pakistan, *** Pakistan Air Force Hospital, Jacobabad Pakistan, ${ }^{* * * *}$ Combined Military \\ Hospital Quetta/National University of Medical Sciences (NUMS) Pakistan
}

\begin{abstract}
Objective: To compare isoconazole nitrate versus nystatin for treatment of otomycosis.

Study Design: Quasi experimental study.

Place and Duration of Study: Combined Military Hospital Bahawalpur and Combined Military Hospital Mardan, from Jan to Dec 2018.

Methodology: Total 204 cases fulfilling the inclusion criteria were selected from outpatient department of Combined Military Hospital Bahawalpur and Mardan. They were divided into 2 groups of 102 each using random number tables. Group A cases were given Isocona-zole nitrate for application in external auditory meatus and group B cases were given Nystatin. All the cases were examined two weeks later and outcome was compared in terms of clinical improvement and symptoms resolution. Results: Out of 204, 125 patients were females and rest 79 were males. Age range was from $12-87$ years with mean age $31 \pm 2.4$. Both groups were identical in terms of gender. In group A patients, 74 (72.5\%) showed marked improvement after two week, $15(14.7 \%)$ showed moderate improvement and $13(12.7 \%)$ showed minor improvement. However, in group B, 55 (53.9\%) showed marked improvement after two week, 23 (22.5\%) showed moderate improvement and 24 (23.5\%) showed minor improvement. Group A treatment was found significantly better than group B treatment $(p=0.021)$.

Conclusion: Isoconazole nitrate ointment was found significantly more effective for treating otomycosis than nystatin.
\end{abstract}

Keywords: Otomycosis, Isoconazole, Nystatin.

How to Cite This Article: Khan MA, Anwar B, Akram S, Ahmed A, Akhtar S. Comparison of Isoconazole Nitrate Versus Nystatin for Treatment of Otomycosis. Pak Armed Forces Med J 2021; 71 (Suppl-3): S581-584. Doi: https://doi.org/10.51253/pafmj.v1i1.7949

\footnotetext{
This is an Open Access article distributed under the terms of the Creative Commons Attribution License (https://creativecommons.org/licenses/by-nc/4.0/), which permits unrestricted use, distribution, and reproduction in any medium, provided the original work is properly cited.
}

\section{INTRODUCTION}

Otomycosis is known by common name of ear fungal infection which refers to a condition that has been described as superficial fungal infection of ear canal. It is also called as fungal otitis externa. Prevalence of otomycosis is around $7 \%$ of the individuals who report with otitis externa. ${ }^{1}$ The causative agents in otomycosis are Aspergillus and Candida. About 80\% of otomycosis is caused by Aspergillus while that of Candida is the second most leading cause of otomycosis. ${ }^{2}$ The geographical area where otomycosis is found to occur mostly is tropical and subtropical areas due to hot, dusty and humid climate. ${ }^{3}$ Frequent swimming, use of ear swabs, scratching the external auditory canal with non-sterile equipments, presence of cerumen and irrational use of topical steroids/antibiotic drops all are contributing factors for the occurrence of otomycosis. ${ }^{4}$ It affects all age groups and both genders. Females are affected more than males. Rate of occurrence of otomycosis is more in females as compared to males. ${ }^{5}$

Signs and symptoms associated with otomycosis

Correspondence: Dr Muhammad Ahmed Khan, Department of ENT, Combined Military Hospital Mardan-Pakistan include pain, itching, inflammation, flaky skin, swelling, redness, ear buzzing, feeling of fullness in the ear, discharge of fluid from the ears and hearing problems. ${ }^{5}$ There are various treatment options to treat otomycosis but it can become chronic so longer treatment and follow up is required). In our setups, cases of otomycosis are clinically diagnosed and rarely culture sensitivity or lab diagnosis via $\mathrm{KOH}$ (potassium hydroxide) etc is required. There are two believes about treatment of otomycosis, one group believes in species identification and susceptibility, ${ }^{6}$ while another group believes that treatment is independent of causative organism rather it depends upon efficacy of drug. ${ }^{7}$ Despite of long term treatment and follow up, the rate of reoccurrence is still very high that's why it may present challenges both to the patient and ENT specialist. In vivo and in vitro studies for otomycosis were conducted by many authors. Three different agents such as antifungal, antimicrobials and keratolytics are used. ${ }^{8}$ Effective treatment regimen is yet to be decided although newer and efficacious drugs have been in use. Eliminating the susceptible factor by use of antifungal agents and removal of ear debris is a common practice. Antifungal agents do not necessary always cure the 
disease. ${ }^{9}$ As otomycosis has higher reoccurrence rate so it should be treated promptly. For the control of this condition many topical agents are used. Most common antifungal agents used include Nystatin, azole group, aluminium acetate drops, boric acid, salicylic acid etc. Antifungal ointments have benefit of less local irritation and prolonged period of contact with affected part of ear.

The objective of our study was to compare two common locally applied antifungal agents in order to find out the more appropriate and effective treatment regimen for otomycosis.

\section{METHODOLOGY}

The quasi-experimental study was carried out at Combined Military Hospital Bahawalpur and Mardan, from January to December 2018. Sample size was calculated from online Rao Software taking 7\% prevalence for otomycosis. ${ }^{1}$ Margin of error was $5 \%$ and confidence interval (CI) $95 \%$. Sample size came to be 100 . Sampling technique used was non-probability convenient sampling. We took 102 cases in each group. Two extra cases were allotted in each group to cater for any cases who may lose to follow up. The cases were selected from ENT outpatient department by otorhinolaryngologists of Combined Military Hospital Mardan and Bahawalpur. Diagnosis of otomycosis was made on the criteria mentioned below.

Symptoms including itching, pain, deafness, blocked ear, tinnitus and otoscopic findings showing hyphae, spores or curdlike white or grey discharge in external auditory meatus of the patient. Presence of fungal elements in $10 \%$ potassium hydroxide $(\mathrm{KOH})$ methylene blue preparation.

Inclusion Criteria: Positive culture of fungal material (discharge, mould etc). Inclusion criteria was patients of both gender and any age who were diagnosed as cases of otomycosis. Clinical diagnosis along with confirmation with potassium hydroxide $(\mathrm{KOH})$ preparation or positive culture was deemed sufficient for inclusion in subject study.

Exclusion Criteria: was patients suffering from diabetes mellitus, chronic liver/renal diseases, tuberculosis, syphilis, immunocompromised individuals or using immunosuppressants, who had recently used any treatment for otomycosis, those who were sensitive to the medicines used in our study i.e. Isoconazole and Nystatin. Those who had concomitant otitis media or fungal infection on any other part of body i.e. tenia corporis etc were also excluded.
All the cases fulfilling the criteria were selected from outpatient department. Proper thorough history was taken for symptoms and use of ear wooden sticks, ear plugs, history of wax cleansing/era trauma, use of local or systemic antibiotics/steroids etc. Other fungal infections of body were also ruled out after examination. Fungal infections of other parts of body eg nails, scalp, vagina etc can cause seeding of infections through scratching and transfer of fungal elements into external auditory meatus. In such cases of seeding or transfer of fungal infections from other parts of body, fungal infection of other parts needs to be treated simultaneously for proper eradication of fungal infection.

After selection cases were divided into two groups, group A and group B randomly through random number tables. Each group had 102 cases. Written consent was taken from all cases. No objection certificate was obtained from ethical committee of hospital for subject research. Group A cases were treated by Isoconazole nitrate ointment and Group B were treated by Nystatin ointment. All the patients were instructed how to apply the oinments in the external auditory meatus. The cases were practically demonstrated the procedure of ointment application. The patients were assessed 2 week after the treatment and the outcome was assessed in terms of improvement of symptoms (reduction in pain, itching and discharge). Clinically improvement was checked on ear examination. Reduction in swelling of ear canal, reduction in discharge, decreased debris and pain/tenderness were signs of clinical improvement. Improvement was categorized into mild, moderate and marked improvement which was assessed clinically and upon asking the individuals about symptoms resolution. Improvement was termed as mild when there was only mild decrease in severity of symptoms i.e., pain, itching, discharge, ear bloackage etc. Similarly improvement was termed moderate and marked upon moderate and marked reduction in severity of symptoms.

All the data was analyzed through SPSS-20. Mean \pm SD was reported for quantitative variables. Frequencies and percentages were calculated for quantitative variables. Chi square was used to compare qualitative outcome i.e. improvement between twogroups. $p$-value $<0.05$ was considered significant. No objection certificate was obtained from Institution Ethical Review Board for this study. Informed consent was taken from all the cases. 


\section{RESULTS}

There were 204 patients selected from ENT outpatient department for subject study (102 cases in each group). Out of 204, 125 (61.3\%) patients were females and rest $79(38.7 \%)$ were males. In group A, there were 64 females $(62.7 \%)$ and 38 males $(37.3 \%)$. In group B, there were 61 females (59.8\%) and 41 males (40.2\%). Age range was from 12 to 87 yearswith mean age $31 \pm$ 2.4. Both groups were identical in terms of gender as shown.

In group A patients, 74 (72.5\%) showed marked improvement after two week, 15 (14.7\%) showed moderate improvement and $13(12.7 \%)$ showed minor improvement. However in group B, 55 (53.9\%) showed marked improvement after two week, 23 (22.5\%) showed moderate improvement and 24 (23.5\%) showed minor improvement. Group A treatment had significantly better outcome as compared to group B i.e., Itraconazole was better than Nystatin $(p=0.021)$ as shown in Table. does the cases of otomycosis. Hot and humid environment favors the growth of fungi.

External auditary meatus is covered by skin on its outer part. This skin resembles skin of rest of the body but it is narrowed in form of a tube, meatal inlet. It can provide conditions for fungal growth. Presence of additional aberrations like narrowing of external auditary meatus further predispose. Manipulation of auditory meatus with hard objects like pins, keys, sticks and other metallic objects is an important predisposing factor. Frequent excessive rubbing of ear to relieve itch is also common. All the above practices can cause ear trauma and predispose to otomycosis. Hot, humid, and acidic $\mathrm{pH}$ local environment in presence of all above predisposing factor favor growth of fungi and cause otomycosis. Onychomycosis and fungal infection of other parts of body cause transfer of infection to ear through scratching. In such cases, otomycosis has to be treated alongwith fungal infection of primary or other sites of body otherwise chronicity or recurrent infec-

Table: Comparison of improvement between two groups.

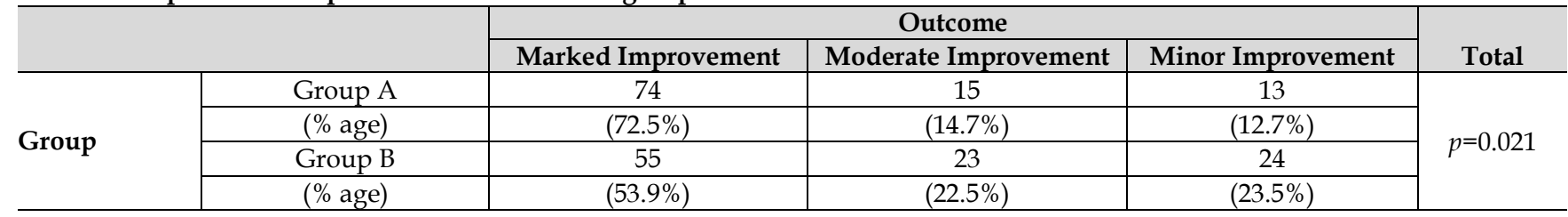

\section{DISCUSSION}

Otomycosis is a common disease seen in ENT outpatient department. There was female predominance in our study which correlates with other studies. ${ }^{10}$ In our study there were $61.27 \%$ females compared to $38.73 \%$ males. Mufattah et al had shown $42.15 \%$ males compared to $57.85 \%$ females. ${ }^{10}$

The incidence of otomycosis is reported to be high in countries on or near tropics. ${ }^{10}$ This study has been carried out in Mardan and Bahawalpur. Mardan district (located in KPK province) has long, humid and sweltering summers with temperature varying from $39-105 \mathrm{~F}$, rarely below $34 \mathrm{~F}$ or above $112 \mathrm{~F}$. Hot summer season lasts from May to September. However winter season lasts only 4 months i.e from November to start of March. Mardan experiences humid season from June to September. It is because of this hot and humid weather that there is hike in cases of otomycosis during this season in Mardan. Bahawalpur is located in southern Punjab. The summers are hot and dry but during July to Septembers humidity increases and so tions are expected.

Controversy exists between the types of antifungal ointment to be prescribed by otorhinolaryngologists. ${ }^{11}$ We have compared nystatin versus isoconazole in present study. There is no previous study which has particularly compared these two antifungals. Previously, Navaneethan et al had compared Fluconazole, Miconazole and Clotrimazole in their study carried out in 2015 on cases of otomycosis in terms of empirical treatment. ${ }^{12}$

In common routine practice, culture \& sensitivity of mycological sample is not routinely carried out because of socioeconomic factors and delay involved. Unless, individual is immunocompromised (severe diabetic, on high dose corticosteroids, taking immunosuppressant, or suffering from HIV or Tuberculosis etc), there are rarely other species of fungi involved other than common Candida or aspergillus. ${ }^{13,14}$ Nystatin and Isoconazole both have adequate activity against Aspergillus and Candida fungi. Nystatin belongs to group of Ionophores, resembling Amphotericin B. It exerts its antifungal action by attaching to ergosterol, 
an important component of fungus membrane. It causes pores in the membrane of fungus which results in leakage of potassium and death of fungus. On the other hand, isoconazole nitrate is an azole derivative. Isoconazole nitrate causes damage to fungus cell through reduction in ATP concentration and damage to its cell wall. Isoconazole nitrate is a broad spectrum antimycotic drug which is not only effective antimycotic but also contains activity against certain gram positive bacteria. It has rapid absorption rate with minimal systemic side effects. Veraldi et al has shown isoconazole to have faster onset of action, faster relief of itching and anti-inflammatory action, impro-ved overall therapeutic efficacy. ${ }^{15,16}$

In vivo and vitro studies have shown azoles to be more effective against common skin and ear fungal infections than Nystatin. ${ }^{17,18}$ Same was observed in our study, where we found better recovery rates/improvement in clinical condition with Isoconazole ointment as compared to Nystatin ointment. Thus we suggest that Isoconazole should be used as first line empirical treatment for otomycosis in immunocompetent individuals as compared to Nystatin.

\section{CONCLUSION}

Isoconazole nitrate ointment was significantly more effective for treating otomycosis than nystatin.

\section{Conflict of Interest: None.}

\section{Authors' Contribution}

MAK: Direct Contribution, BA: Script Writing, SA: Data Analysis AA: Data Collection, Review, SA: Data analysis.

\section{REFERENCES}

1. Anwar k, Gohar MS. Otomycosis; Clinical features, predisposing factors and treatment implications. Pak J Med Sci 2014; 30(3): 564-567.

2. Dundar R, Iynen I. Single dose topical treatment of clotrimazole for the treatment of otomycosis: Is it enough?. J Audiol Otol 2018; 23(1): 15-19.
3. Munguia R, Daniel JS. Ototopical antifungals and otomycosis. Int J Pediatr Otorhinolaryngol 2008; 72(4): 453-459.

4. Mahmoudabadi AZ. Mycological studies in 15 cases of otomycosis. Pak J Med Sci 2006; 22(4): 486-488.

5. Kurnatowski P, Filipiak A. Otomycosis: prevalence, clinical symptoms, therapeutic procedure. ycoses 2001; 44(11-12): 472-479.

6. Arthur RR, Drew RH, Perfect JR. Novel modes of antifungal drug administration. Expert Opin Investig Drugs 2004; 13(8): 903-932.

7. Araiza J, Canseco P, Bonifaz A. Otomycosis: clinical and mycological study of 97 cases. Rev Laryngol Otol Rhinol (Bord) 2006; 127(4): 251-254.

8. Blyth CC, Palasanthiran P, O'Brien TA. Antifungal therapy in children with invasive fungal infections: a systematic review. Pediat 2007; 119(4): 772-784.

9. Mishra N, Rastogi MK, Gahalaut P, Yadav S, Srivastava N, Aggarval A. Clinicomycological study of dermaophytosis in children: Ind J Pediat Dermatol 2018; 19(1): 326-330.

10. Mofatteh MR, Yazdi ZN, Yousefi M, Namaei MH. Comparison of recovery rates of otomycosis using betadine and clotrimazole topical treatment. Braz J otolaryngol 2018; 84(4): 404-409.

11. Ravindran A, sagesh M. Effectiveness of oral itraconazole in management of otomycosis with tympanic membrane perforation. Int J Med Rex Sci 2017; 5(4): 1373-1376.

12. Navaneethan N. Type of antifungals; does it really matter in empirical treatment of otomycosis. Ind J Otolaryngol Head Neck J 2015; 67(1) : 64-67.

13. Hoshino T, Matsumoto M. Otomycosis: subdermal growth in calcified mass. Euro Arch Otorhinolaryngol 2006; 263(9): 875-878.

14. Gutierrez P, Alvarez J, Sanudo E. Presumed diagnosis: otomycosis. a study of 451 patients. Acta otorhnolaryngol Esp 2005; 56(5): 181-186.

15. Veraldi S. Isoconazole nitrate: a unique broad-spectrum antimicrobial azole effective in the treatment of dermatomycoses, both as monotherapy and in combination with corticosteroids. Mycosis 2013; 56 (Suppl-1): 3-15.

16. Heeres J, Meerpoel L, Lewi P. Review Conazoles. Molecul 2010; 15(6): 4129-4188.

17. Agarwal P, Devi LS. Otomycosis in a rural community attending a tertiary care hospital. Assessment of risk factors and identification of fungal and bacterial agents. J Clin Diagn Res 2017; 11(6): DC 14-DC18.

18. Mishra P, Schdeva S, Kaushik M, Agarwal D. Otomycosis treatment: Topical drops versus cream - a prospective randomized study. Arch Otolaryngol Rhinol 2017; 3(3): 106-108. 